\title{
EVIDENCIAS Y RETOS DE LA ATENCIÓN TEMPRANA: EL MODELO CENTRADO EN LA FAMILIA
}

\section{Evidence and Challenges of Early Intervention: the Family-Centered Model}

\author{
Sara BAgur Pons \\ Universidad de las Islas Baleares. Departamento de Pedagogía Aplicada \\ y Psicología de la Educación \\ sarabagpons@gmail.com \\ Sebastià Verger GelaberT \\ Universidad de las Islas Baleares. Departamento de Pedagogía Aplicada \\ y Psicología de la Educación
}

Recepción: 22 de enero de 2020

Aceptación definitiva: 17 de julio de 2020

Resumen: El propósito de la Atención Temprana es ofrecer un servicio de calidad para el máximo desarrollo y autonomía de los niños y abogar por la calidad de vida y el bienestar familiar. El modelo actual de intervención se identifica con las prácticas centradas en la familia, basadas en la construcción de capacidades familiares, donde los profesionales deben actuar como colaboradores para dar respuesta a las necesidades de los progenitores. Este artículo pretende realizar una revisión sistematizada de la literatura, para analizar las investigaciones empíricas en el campo de la Atención Temprana desde el año 2000 e identificar aspectos que inducen a nuevas investigaciones. Se hallaron y analizaron un total de 33 artículos. Los resultados muestran que, a pesar de que se lleve a cabo el modelo centrado en la familia, hay obstáculos para desempeñar prácticas participativas, ya que implican la modificación de los apoyos profesionales y, al mismo tiempo, cambios en el protagonismo de la familia durante la intervención. La formación, la actitud del profesional o la toma de decisiones conjunta entre el profesional y la familia son aspectos que requieren ser mejorados de acuerdo con los principios del modelo centrado en la familia. Además, se precisa mayor investigación en la relación profesional-familia, el bienestar familiar, el desarrollo de nuevos programas y la actitud de los profesionales para consagrar la calidad de los servicios de Atención Temprana.

Palabras clave: Atención Temprana; familia; desarrollo profesional; calidad del servicio. 
АвsтRACт: The purpose of Early Childhood Intervention is to provide a quality service for the maximum development and autonomy of children and to advocate quality of life and family well-being. The current model of intervention is focused on family-centred practices. These are based on building family capacity, where professionals must act as partners to respond to parents' needs. This article aims to conduct a systematized review of the literature, to analyze empirical research in the field of Early Intervention since 2000 and to identify aspects that lead to new research. A total of 33 articles were analysed and percived. The results show that despite family-centred practices, there are obstacles to participatory. These involve the modification of professional supports and, at the same time, the role of the family during the intervention. The training, the attitude of the professional or the joint decision making between the professional and the family are aspects that require improvement according to the principles of the family-centred model. In addition, more research is needed on the professional-family relationship, family welfare, the development of new programs and the attitude of professionals to establish the quality of Early Intervention services.

KEY WORDs: Early Intervention; family; professional development; percieved quality.

\section{Introducción}

L

a Atención Temprana es el campo referente en la prevención e intervención de las alteraciones del desarrollo en la primera infancia, lo cual hace que sea insustituible para el futuro de la sociedad (Millá, 2005). Diferentes disciplinas -Medicina, Neurología, Fisiología, Genética, Psicología, Pedagogía- han ido originando conocimiento, otorgando importancia a la primera infancia como etapa fundamental para el desarrollo de la vida (Gútiez, 2005). Los inicios de la Atención Temprana se remontan al año 1965 en EE. UU. como modelo médico-asistencialista para dar respuesta a las discapacidades de los niños (Díaz, 2019; Rouse, 2012). El principal objetivo era "recuperar o rehabilitar las áreas afectadas" (Romero, Gálvez, Belda y Millá, 2015, p. 72) del desarrollo, dando un papel pasivo al entorno y a la familia, asociando el concepto a estimulación precoz (Belda, 2016; Romero, Barajas y Gálvez, 2015). Este modelo estaba plenamente centrado en el niño, otorgando valía al papel de los profesionales expertos en las diferentes disciplinas (Ponte, Cardama, Arlanzón, Belda, González y Vived, 2004).

Entrada la década de los ochenta, el interés científico en Europa y en Norteamérica ofreció programas relevantes de mejora de los servicios (Romero, Gálvez, Belda y Millá, 2015), potenciando modelos psicopedagógicos para favorecer las posibilidades físicas e intelectuales del niño, cambiando el concepto a estimulación temprana (Gútiez, 2005). Además, la Organización de las Naciones Unidas aprobó los derechos de la infancia en 1989, de modo que se reconsideró el desarrollo pleno de los niños a partir de la responsabilidad colectiva, institucional y ciudadana (ONU, 1989; CERMI, 2017). Así emergió un nuevo paradigma que propugnaba el papel necesario de las familias, donde el equipo multidisciplinar de profesionales debía planificar de acuerdo con las necesidades de los progenitores (Giné, García, Vilaseca y Barcells, 2008). 
Este planteamiento se enmarca en diferentes teorías que ayudaron a impulsar las nuevas prácticas en Atención Temprana. Principalmente, la influencia proviene del modelo ecológico de Bronfenbrenner (1979), el modelo transaccional de Sameroff y Chandler (1975), la teoría de la modificabilidad cognitiva estructural de Feuerstein (1989) y el modelo de desarrollo precoz y factores de riesgo de Guralnick (1998). En gran medida, surgió la idea de que el desarrollo humano se construye a partir de las interacciones que los sujetos mantienen en sus diferentes entornos, directa o indirectamente (Bronfenbrenner, 1987; Sameroff y Fiese, 2000).

En la actualidad, las acciones en el ámbito de la Atención Temprana deben contemplar los entornos naturales para favorecer la máxima inclusión del niño en todos sus contextos (Cañadas, 2012; Martínez y Calet, 2015; Rouse, 2012). Concretamente, se debe entender que la familia "es un sistema complejo con sus propias y únicas características y necesidades” (Leal, 2008, p. 9), de manera que la unidad familiar es mayor que la suma de sus partes y que cualquier vivencia y experiencia de uno de los miembros provoca impactos sobre el resto. Pues el modelo integrador -psicopedagógico, ecológico y transaccional- actúa en tres niveles de prevención para favorecer "la intervención sobre el niño, la familia y el entorno” (Gútiez, 2005, p. 277).

Cabe incidir en la evolución explícita de los modelos de Atención Temprana para conceptualizar el paradigma actual. Dunst, Johanson, Trivette y Hamby (1991) detallan cuatro modelos de intervención (en Espe-Sherwind, 2008, p. 2):

- Modelo centrado en el profesional: el profesional es el experto de la disciplina, el cual expone los objetivos y el programa para satisfacer las necesidades del niño.

- Modelo familiar: tanto los profesionales como las familias intervienen durante el proceso, pese a que los primeros siguen identificando las necesidades a satisfacer.

- Modelo enfocado en la familia: los profesionales perciben a las familias como usuarias y consumidoras del servicio y, con su ayuda, pueden escoger entre diversas opciones de intervención.

- Modelo centrado en la familia: los profesionales y las familias actúan en igualdad de condiciones. Las necesidades son identificadas por los progenitores y la intervención va enfocada a la capacitación y el funcionamiento familiar.

Intervenir a partir del modelo centrado en la familia supone que el rol del profesional sea el de colaborador, movilizando las ayudas necesarias y ejerciendo un equilibrio de poder con la familia (Cañadas, 2012), centrándose en las fortalezas y en la competencia familiar (Leal, 2008; Dempsey y Dunst, 2004; Dempsey y Keen, 2008; Trivette, Dunst y Hamby, 2010).

Actualmente, la Atención Temprana en España es irregular, minoritaria y desigual, sin renovaciones significativas desde la Ley 39/2006, de 14 de diciembre, de Promoción de la Autonomía Personal y Atención a las Personas en situación de Dependencia (CERMI, 2017), habiendo diferencias en el territorio nacional debido a la descentralización política y al desarrollo propio del servicio en cada comunidad autónoma (Romero, Lillo y García, 2018). Sin embargo, el concepto se ha visto 
modificado por la evolución de las experiencias, las buenas prácticas y las investigaciones que se han llevado a cabo en las últimas décadas (Millá, 2005). De este modo, una definición de referencia de la Atención Temprana la identifica como:

El conjunto de intervenciones dirigidas a la población infantil de 0-6 años, a la familia y al entorno, que tienen por objetivo dar respuesta lo más pronto posible a las necesidades transitorias o permanentes que presentan los niños con trastornos en el desarrollo o que tienen riesgo de padecerlos (GAT, 2000, p. 12).

La Atención Temprana de calidad debe permitir el desarrollo y la autonomía de los niños, así como la calidad de vida y el bienestar familiar (CERMI, 2017). El modelo actual se centra en la familia con el objetivo de que los progenitores puedan desarrollar habilidades parentales y participar en la vida de sus hijos (GAT, 2005). Por eso, es fundamental que durante el proceso las familias expresen creencias, sentimientos, expectativas y la historia familiar para que la evaluación, la planificación y la intervención sean efectivas (Giné et al., 2008).

En términos generales, las premisas básicas del modelo centrado en la familia son (Leal, 2008):

- La familia como unidad de apoyo: la familia es el núcleo central de atención teniendo en cuenta sus necesidades y su bienestar (Dunst, 2000).

- La familia escoge: los progenitores tienen voz para exponer sus preferencias y los profesionales deben dar respuesta a estas necesidades. Considerar este punto es uno de los mayores retos, ya que los servicios deberán reflexionar sobre cómo enfocar la participación de los padres y madres y cuál será el propósito de la intervención (Giné et al., 2008).

- Apoyos basados en fortalezas: el objetivo es capacitar-empoderar-a las familias y promover una mejor calidad de vida. Capacitar significa "crear oportunidades para que los miembros sean más competentes, independientes y autosuficientes" (Leal, 2008, p. 49), de manera que los apoyos que se consideran más adecuados son los que implican a los progenitores directamente (Dunst, 2000; Trivette y Dunst, 2004).

Por ello, los profesionales deben abandonar el modelo de experto, otorgando importancia a las actitudes positivas, ya que ayudan a que los progenitores tengan una visión óptima de sus problemas rutinarios y a recuperar la satisfacción y la confianza hacia sus hijos (Giné et al., 2008). Por ende, los equipos de profesionales deben llevar a cabo prácticas de capacitación, las cuales se construyen a partir de (Dempsey y Keen, 2008; Dunst y Trivette, 2009; Trivette y Dunst, 2004):

- Prácticas de relación: basadas en la escucha activa, la empatía, las creencias, los valores, las fortalezas familiares y la comunicación efectiva.

- Prácticas de participación: basadas en el uso de competencias, habilidades familiares y el desarrollo de nuevas capacidades. Implican cambios significativos para los profesionales, ya que suponen modificar los apoyos y la manera de entender la figura de los progenitores como colaboradores del proceso. 
McWilliam (2010), autor referente del modelo centrado en la familia en entornos naturales, propuso el modelo de intervención basado en rutinas, que viene determinado por cinco componentes:

- La ecología familiar: conocer los apoyos naturales de la familia, las personas significativas para el niño y el papel que adoptan durante su vida diaria.

- La evaluación basada en rutinas: determina los objetivos específicos de la intervención. Con la entrevista basada en rutinas se extrae un análisis detallado sobre el funcionamiento del niño y la familia para acordar un plan individual de apoyo familiar (PIAF).

- El profesional de referencia: un solo profesional da apoyo al niño y a la familia durante la intervención, a la vez que él recibe ayuda del equipo de profesionales.

- Visitas efectivas en el hogar: se basan en una planificación que da respuesta a las necesidades para adecuar las rutinas diarias del núcleo familiar, ya que se lleva a cabo en un "espacio para la verdadera colaboración” (Díaz, 2019, p. 118).

- Colaboración y asesoramiento en la escuela: se debe incluir a los profesionales que están en contacto directo en el entorno natural del niño.

Paralelamente, cabe destacar que los Centros de Desarrollo Infantil y Atención Temprana (CDIAT) han ido evolucionado durante más de dos décadas para adecuarse a las demandas de la población infantil. Actualmente, "son centros públicos o subvencionados por recursos públicos que deben ser administrados bajo el principio de eficacia, economía y eficiencia” (Alcantud-Marín, Pérez-Bou y Alonso-Esteban, 2017, p. 55). Los dispositivos multidisciplinares operan como redes de servicios dando orientación, globalidad, efectividad, continuidad y coordinación para atender las necesidades detectadas partiendo de los derechos de las personas (GAT, 2018). Pues no solo se debe considerar el tipo de intervención o tratamiento, sino "realizarlo con la mayor calidad posible” (Millá, 2005, p. 899). Gracias a la aportación de diferentes proyectos (Tabla 1), la calidad de los servicios ha ido mejorando durante las últimas décadas. Tanto la multiprofesionalidad, la estructura, el funcionamiento y la optimización de los recursos, como los aspectos físicos del servicio (accesibilidad, dimensión, salas, equipamiento) y, sobre todo, la metodología de intervención (coordinación, atención secuenciada, actuación globalizada y acción integradora) determinan aspectos referentes a la calidad (Belda, 2016; GAT, 2005; Millá, 2005).

Existen dos modelos que configuran los grupos de profesionales: el equipo interdisciplinar, formado por profesionales especialistas en diferentes disciplinas que comparten información y actúan de forma conjunta; y el equipo transdisciplinar, formado por profesionales que adquieren conocimiento en diversas disciplinas y las incorporan en su formación inicial. En este segundo canon, un profesional debe asumir la responsabilidad de atender al niño y a su familia (GAT, 2000), de manera que el $75 \%$ del tiempo debe apoyar a la unidad familiar y el $25 \%$ dar soporte al equipo de profesionales (McWilliam, 2010).

Los CDIAT deben abogar por "una propuesta comprensiva, integradora, de atención y apoyos basados y centrados en” (Tamarit, 2015, p. 60):

- El conocimiento sobre el desarrollo humano y sobre sus alteraciones. 


\begin{tabular}{|l|c|c|}
\hline \multicolumn{2}{|c|}{ Tabla 1. Documentos nacionales de Atención Temprana } \\
\hline \multicolumn{1}{|c|}{ Editor } & Año \\
\hline Criterios de Calidad en Centros de Atención Temprana. & Grupo PADI & 1996 \\
\hline Manual de Buenas Prácticas de Atención Temprana. & FEAPS & 1999 \\
\hline Libro Blanco de la Atención Temprana. & GAT & 2000 \\
\hline Atención Temprana. Orientaciones para la calidad. & FEAPS & 2001 \\
\hline $\begin{array}{l}\text { Programa INTER. Guía de estándares de calidad de la Aten- } \\
\text { ción Temprana. }\end{array}$ & IMSERSO & 2004 \\
\hline Organización Diagnóstica para la Atención Temprana. & GAT & 2005 \\
\hline $\begin{array}{l}\text { Recomendaciones técnicas para el desarrollo de la Atención } \\
\text { Temprana. }\end{array}$ & GAT & 2005 \\
\hline $\begin{array}{l}\text { La Atención Temprana en España. } \\
\text { Real Patronato }\end{array}$ & 2011 \\
\hline La realidad actual de la Atención Temprana en España. & GAT & 2011 \\
\hline $\begin{array}{l}\text { 10 años comprometidos con la excelencia. Buenas prácticas de } \\
\text { calidad FEAPS (V). }\end{array}$ & Plena Inclusión & 2014 \\
\hline Atención Temprana la visión de los profesionales. & GAT & 2019 \\
\hline Guía Básica sobre Atención Temprana y Transformación. & Plena Inclusión & 2019 \\
\hline
\end{tabular}

Fuente: Elaboración propia.

- El uso de prácticas basadas en la evidencia y en la ética.

- Enfoques centrados en la familia (incluido el niño).

- El logro de mayor bienestar familiar.

- El logro de mayor bienestar y desarrollo personal.

- La intervención en el contexto natural.

- Acciones para contribuir a la generación de contextos protectores e inclusivos.

\section{Método}

El estudio pretende realizar una revisión sistematizada de la literatura para analizar las investigaciones empíricas en el campo de la Atención Temprana desde el año 2000 e identificar aspectos que inducen a nuevas investigaciones. En el campo de las Ciencias Sociales, la sistematización promueve el análisis y la exploración de las áreas de conocimiento donde el foco de atención recae en "la identificación de las tendencias y corrientes principales [...], así como la detección de huecos y oportunidades de investigación" (Codina, 2018, p. 11), promoviendo la transparencia y el rigor del estudio (Hart, 2008). A modo general, se buscaron los documentos en bases de datos específicos, se filtraron y seleccionaron los documentos a partir de criterios claramente definidos y se agruparon, evaluaron y analizaron para la construcción de resultados. 


\subsection{Búsqueda de documentos y filtración de resultados}

La búsqueda documental sobre la evidencia científica se vertebró a partir de ecuaciones de búsqueda en tres bases de datos (Tabla 2): Scopus, Web of Science y Dialent Plus, siguiendo tres fases: facetación, derivación y combinación de las palabras clave. Posteriormente, se analizaron los resultados obtenidos de la base de datos Scopus con la herramienta VOSViewer ${ }^{1}$ que permite la visualización de redes bibliométricas a partir de la matriz de ocurrencia y coocurrencia. En este caso, se dictaminó que el mínimo número de ocurrencias fuera superior o igual a cinco $[f(K W)=5]$, con el objetivo de analizar la frecuencia, detectar los clústeres y determinar las ocurrencias de las palabras clave de los documentos.

\section{TABLA 2. Ecuaciones de búsqueda}

\begin{tabular}{|c|c|c|c|}
\hline Base de datos & Ecuación de búsqueda & Resultados & Fecha \\
\hline Scopus & $\begin{array}{l}\text { ("early child" intervention" OR "early interven- } \\
\text { tion" OR "early attention" OR "early inter- } \\
\text { vention child*") AND ("family-centred practi- } \\
\text { ces" OR "family-centred services" OR "natural } \\
\text { enviroments" OR "traditional model" OR "fa- } \\
\text { mily support" OR "family assessment") AND } \\
\text { (model OR program or intervention) }\end{array}$ & 602 & $28 / 10 / 2019$ \\
\hline WoS & $\begin{array}{l}\text { ("early child" intervention" OR “early interven- } \\
\text { tion children") AND (model OR intervention) } \\
\text { NOT (adolescent OR juv* OR school OR pshi- } \\
\text { cosis) }\end{array}$ & 144 & $30 / 10 / 2019$ \\
\hline Dialnet Plus & $\begin{array}{l}\text { (“atención temprana” OR “intervención tem- } \\
\text { prana”) AND (“modelo" OR “programa” OR } \\
\text { "intervención”) NOT (“joven” OR “adolescen- } \\
\text { cia” OR “escuela” OR “pediatria”) }\end{array}$ & 157 & $31 / 10 / 2019$ \\
\hline & Total & 903 & $31 / 10 / 2019$ \\
\hline
\end{tabular}

Fuente: Elaboración propia.

Así se encontraron cinco clústeres determinados por los colores del mapa de relaciones de la Figura 1, donde la dimensión de los círculos y de la etiqueta de los ítems especifican el peso dentro del clúster. Se identificó que, para elaborar los resultados, se requerían los términos del primer, tercer y cuarto clúster, debido a que representaban alto grado de significatividad de ocurrencias en la red de asociaciones. Esto proporcionó la evaluación positiva y significativa de la búsqueda para la identificación de palabras clave de los documentos procedentes de la base de datos.

1 Creado por Centre for Science and Technology Studies. 


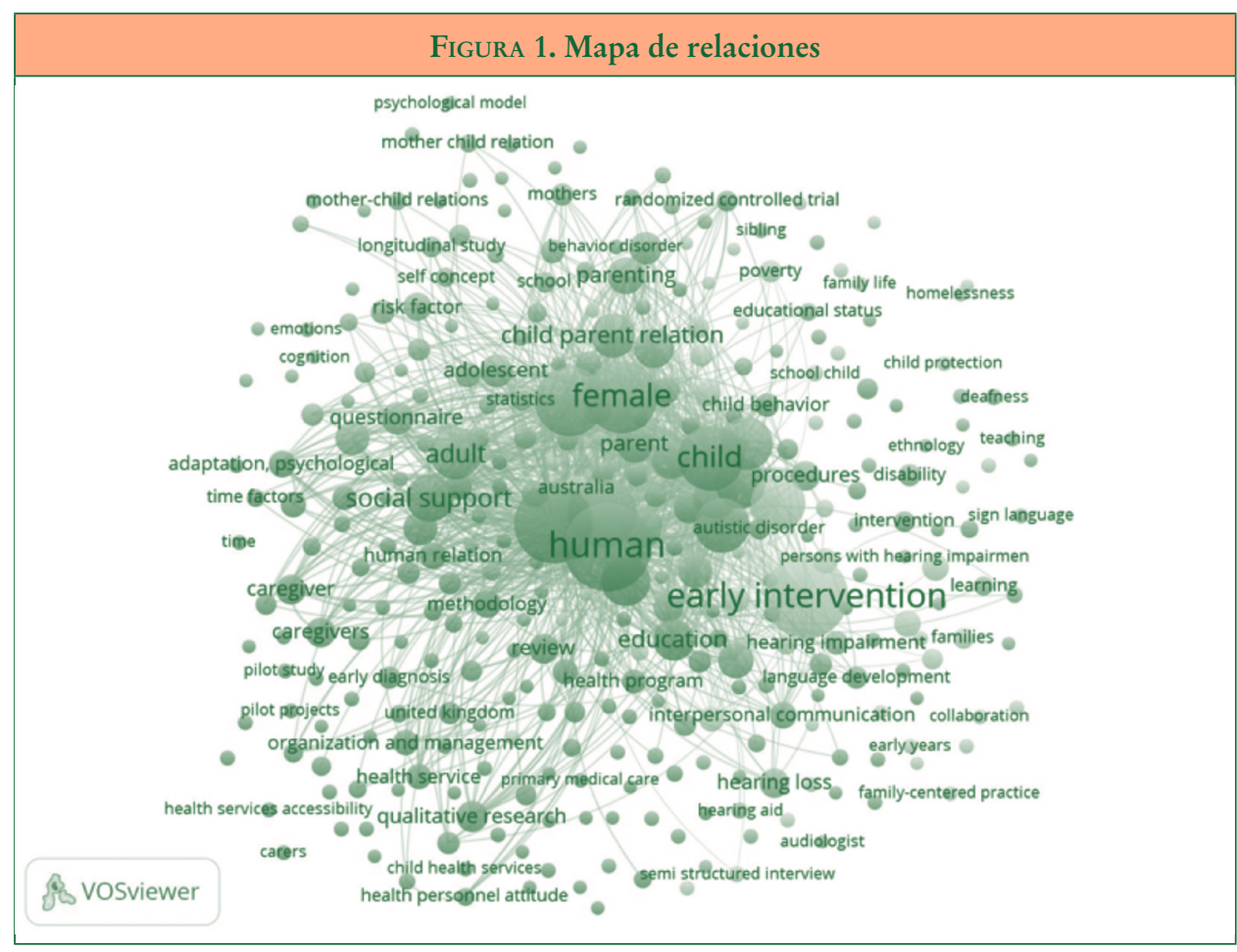

TABLA 3. Criterios de búsqueda documental

\begin{tabular}{|c|c|c|}
\hline Bases de datos & Criterios de selección & Resultados \\
\hline \multirow{4}{*}{ Scopus } & Ubicación palabras clave: título, resumen y palabras clave & \multirow{4}{*}{602} \\
\hline & Lengua: inglés y castellano & \\
\hline & Limitado por campo de estudio & \\
\hline & Año de publicación mayor o igual al 2000 & \\
\hline \multirow{4}{*}{ WoS } & Ubicación palabras clave: título & \multirow{4}{*}{144} \\
\hline & Lengua: inglés y castellano & \\
\hline & Limitado por campo de estudio & \\
\hline & Año de publicación mayor o igual al 2000 & \\
\hline \multirow{5}{*}{ Dialnet Plus } & Ubicación palabras clave: título & \multirow{5}{*}{157} \\
\hline & Lengua: castellano & \\
\hline & No se limita el campo de estudio & \\
\hline & Ubicación de publicación: España & \\
\hline & Año de publicación mayor o igual al 2000 & \\
\hline
\end{tabular}

Fuente: Elaboración propia.

Ediciones Universidad de Salamanca / CC BY-NC-ND

Siglo Cero, vol. 51 (4), 2020, octubre-diciembre, pp. 69-92 
Finalmente, se elaboró la fase de evaluación y, partiendo de los criterios de calidad, se aplicaron determinados criterios de inclusión -intervalo de publicación a partir del año 2000, estudios evaluados por blind peer review, documentos con metodología explícita, documentos escritos en inglés y castellano- (Tabla 3) y criterios de exclusión -duplicación de documentos, número de citas recibidas y campo de estudio- (Figura 2). Cabe destacar que Scopus es la única de las tres bases de datos que proporciona la opción de ubicar la búsqueda de las palabras clave en el título,

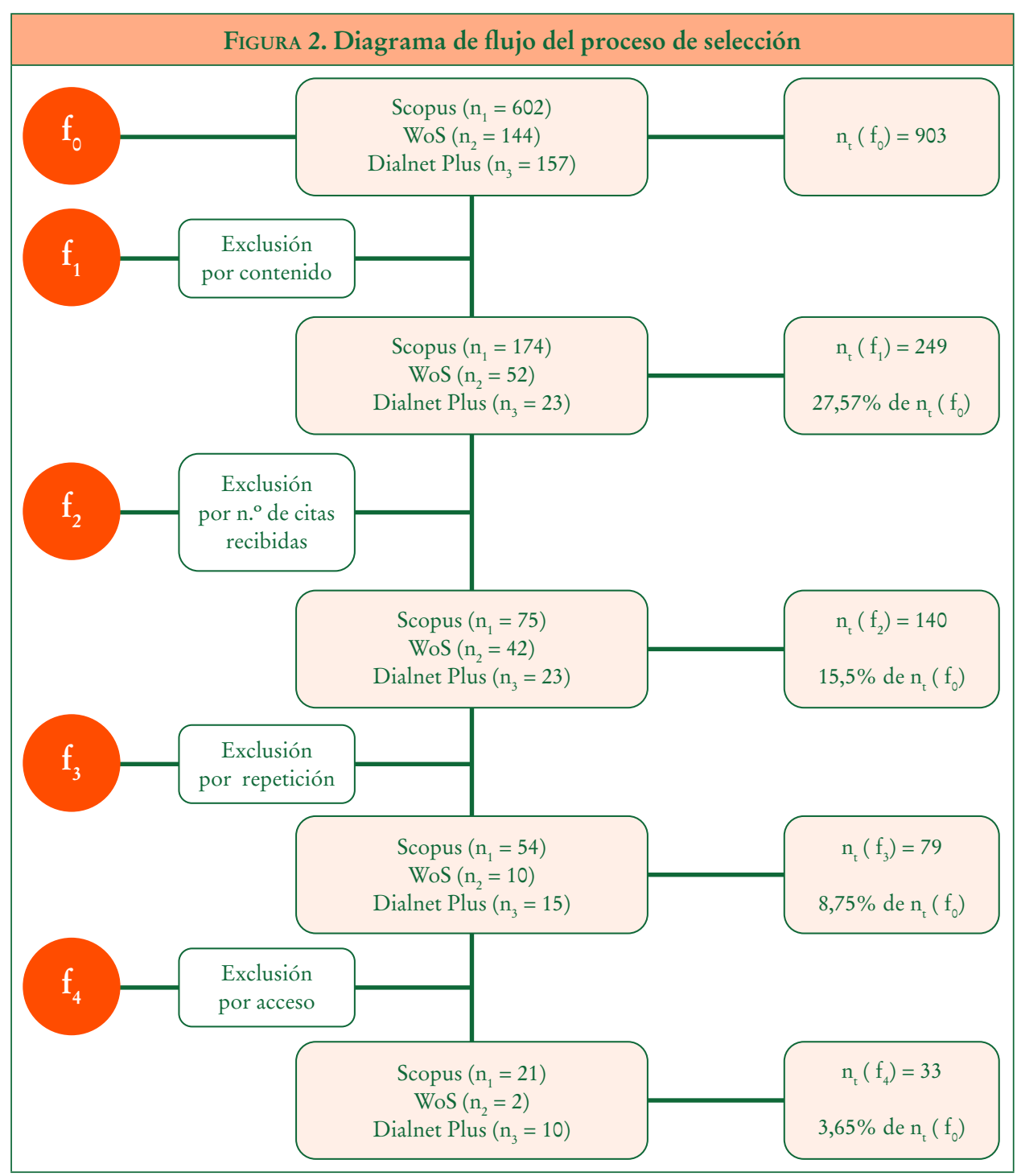

Ediciones Universidad de Salamanca / CC BY-NC-ND

Siglo Cero, vol. 51 (4), 2020, octubre-diciembre, pp. 69-92 
el resumen y las palabras clave de los documentos de manera simultánea. Por lo que hace a la fecha de publicación, en una revisión previa de la literatura se observó que, si se utilizaban intervalos de publicación recientes, por ejemplo en los últimos diez años, los resultados excluían los modelos tradicionales de Atención Temprana, por lo que se determinó ampliar el año de publicación en inglés al 2000. Además, los estudios publicados en español se acotaron al mismo año, debido a la publicación del Libro Blanco de Atención Temprana (GAT, 2000) como documento de referencia nacional.

\subsection{Análisis documental: categorización de documentos e identificación de categorias relacionales}

Esta fase tuvo como objetivo elaborar un análisis de cada uno de los 33 artículos que forman parte de la selección (Tabla 4). El $54 \%$ fueron estudios elaborados en España, el $25 \%$ en Australia, el 14 \% en EE. UU. y el $7 \%$ restante en Portugal. Por otra parte, el $41 \%$ de los estudios utilizan metodología cuantitativa, el $32 \%$ se asocia a la cualitativa y el $27 \%$ a revisiones de la literatura.

\begin{tabular}{|c|c|c|c|}
\hline \multicolumn{4}{|c|}{ TABLA 4. Características de la selección de documentos } \\
\hline Autores, año & Título & País & Met. \\
\hline $\begin{array}{l}\text { Balcells-Balcells, A., Giné, } \\
\text { C., Guàrdia-Olmos, J.y } \\
\text { Summers, J. A. (2016). }\end{array}$ & $\begin{array}{l}\text { Proposal of indexes to evaluate Family Quality of Life, } \\
\text { Partnership, and Family support needs. }\end{array}$ & ESP & CT \\
\hline $\begin{array}{l}\text { Balcells-Balcells, A., Giné, } \\
\text { C., Guàrdia-Olmos, J., } \\
\text { Summers, J. A. y Mas, J. M. } \\
\text { (2019). }\end{array}$ & Impact of supports and partnership on family quality of life. & ESP & CT \\
\hline $\begin{array}{l}\text { Botana, I. y Peralbo, M. } \\
\text { (2014). }\end{array}$ & Familia, estrés y atención temprana. & ESP & CT \\
\hline Cañadas, M. (2012). & $\begin{array}{l}\text { La familia, principal protagonista de los centros de desarrollo } \\
\text { infantil y Atención Temprana. }\end{array}$ & ESP & RL \\
\hline $\begin{array}{l}\text { Cumming, T. y Wong, S. } \\
\text { (2012). }\end{array}$ & $\begin{array}{l}\text { Professionals don't play: challenges for early childhood } \\
\text { educators working in a transdisciplinary early intervention } \\
\text { team. }\end{array}$ & AUS & $\mathrm{CL}$ \\
\hline $\begin{array}{l}\text { Dias, P. C. y Cadime, I. } \\
\text { (2019). }\end{array}$ & $\begin{array}{l}\text { Child and Family-Centered Practices in Early Childhood } \\
\text { Education and Care Services: an empirical study with families } \\
\text { and practitioners in Portugal. }\end{array}$ & PRT & CT \\
\hline $\begin{array}{l}\text { Davis, K. y Gavidia-Payne, } \\
\text { S. (2009). }\end{array}$ & $\begin{array}{l}\text { The impact of child, family, and professional support } \\
\text { characteristics on the quality of life in families of young } \\
\text { children with disabilities. }\end{array}$ & AUS & CT \\
\hline $\begin{array}{l}\text { Dempsey, I. y Dunst, C. J. } \\
\text { (2004). }\end{array}$ & $\begin{array}{l}\text { Helpgiving styles and parent empowerment in families with } \\
\text { a young child with a disability. }\end{array}$ & EE. UU. & $\mathrm{CL}$ \\
\hline
\end{tabular}




\section{TABla 4. Características de la selección de documentos (cont.)}

\begin{tabular}{|c|c|c|c|}
\hline Autores, año & Título & País & Met. \\
\hline $\begin{array}{l}\text { Dunst, C. J., Espe- } \\
\text { Sherwindt, M. y Hamby, } \\
\text { D. W. (2019). }\end{array}$ & $\begin{array}{l}\text { Does capacity-building professional development engender } \\
\text { practitioners' use of capacity-building family-centered } \\
\text { practices? }\end{array}$ & EE. UU. & CT \\
\hline $\begin{array}{l}\text { Dunst, C. J. y Trivette, } \\
\text { C. M. (2009). }\end{array}$ & Capacity-building family-systems intervention practices. & EE. UU. & $\mathrm{RL}$ \\
\hline $\begin{array}{l}\text { Epley, P., Summers, J. A. y } \\
\text { Turnbull, A. (2010). }\end{array}$ & $\begin{array}{l}\text { Characteristics and trends in family-centered } \\
\text { conceptualizations. }\end{array}$ & EE. UU. & $\mathrm{RL}$ \\
\hline $\begin{array}{l}\text { Escorcia-Mora, C. T., } \\
\text { García-Sánchez, F. A., } \\
\text { Sánchez-López, M. C. } \\
\text { y Hernández-Pérez, E. } \\
\text { (2016). }\end{array}$ & $\begin{array}{l}\text { Styles questionnaire of interaction between parents } \\
\text { and practitioners in early intervention: content validity } \\
\text { [Cuestionario de estilos de interacción entre padres y } \\
\text { profesionales en atención temprana: validez de contenido]. }\end{array}$ & ESP & CT \\
\hline $\begin{array}{l}\text { Escorcia-Mora, C. T., } \\
\text { García-Sánchez, F. A., } \\
\text { Sánchez-López, M. C., } \\
\text { Orcajada, N. y Hernández- } \\
\text { Pérez, E. (2018). }\end{array}$ & $\begin{array}{l}\text { Early childhood intervention practices in the southeast of } \\
\text { Spain: professionals and families perspective [Prácticas } \\
\text { de atención temprana en el sureste de España: perspectiva } \\
\text { de profesionales y familias]. }\end{array}$ & ESP & CT \\
\hline Espe-Sherwindt, M. (2008). & $\begin{array}{l}\text { Family-centred practice: collaboration, competency and } \\
\text { evidence. }\end{array}$ & EE. UU. & $\mathrm{RL}$ \\
\hline Evans, S. (2018). & $\begin{array}{l}\text { The social worker as transdisciplinary professional: } \\
\text { a reflective account. }\end{array}$ & AUS & $\mathrm{RL}$ \\
\hline $\begin{array}{l}\text { Fernández, R., Serrano, } \\
\text { A. M., McWilliam, R. y } \\
\text { Cañadas, M. (2017). }\end{array}$ & $\begin{array}{l}\text { Relación entre empoderamiento familiar y calidad de los } \\
\text { servicios de atención temprana. }\end{array}$ & ESP & CT \\
\hline $\begin{array}{l}\text { Fingerhut, P. E., Piro, J., } \\
\text { Sutton, A., Campbell, R., } \\
\text { Lewis, C., Lawji, D. y } \\
\text { Martínez, N. (2013). }\end{array}$ & $\begin{array}{l}\text { Family-centered principles implemented in home-based, } \\
\text { clinic-based, and school-based pediatric settings. }\end{array}$ & EE. UU. & $\mathrm{CL}$ \\
\hline $\begin{array}{l}\text { Fox, G. L., Nordquist, } \\
\text { V. M., Billen, R. M. y } \\
\text { Savoca, E. F. (2015). }\end{array}$ & $\begin{array}{l}\text { Father involvement and Early Intervention: effects } \\
\text { of empowerment and father role identity. }\end{array}$ & EE. UU. & $\mathrm{CL}$ \\
\hline $\begin{array}{l}\text { García-Sánchez, F. A., } \\
\text { Rubio-Gómez, N., } \\
\text { Orcajada-Sánchez, N., } \\
\text { Escorcia-Mora, C. T. y } \\
\text { Cañadas, M. (2018). }\end{array}$ & $\begin{array}{l}\text { Training needs in family-centered practices for Spanish } \\
\text { early childhood intervention professionals [Necesidades de } \\
\text { formación en prácticas centradas en la familia en profesionales } \\
\text { de atención temprana españoles]. }\end{array}$ & ESP & CT \\
\hline $\begin{array}{l}\text { Gavidia-Payne, S., Meddis, } \\
\text { K. y Mahar, N. (2015). }\end{array}$ & $\begin{array}{l}\text { Correlates of child and family outcomes in an Australian } \\
\text { communitybased early childhood intervention program. }\end{array}$ & AUS & $\mathrm{CL}$ \\
\hline $\begin{array}{l}\text { Hughes-Scholes, C. H. y } \\
\text { Gavidia-Payne, S. (2019). }\end{array}$ & $\begin{array}{l}\text { Early Childhood Intervention Program Quality: examining } \\
\text { family-centered practice, parental self-efficacy and child and } \\
\text { family outcomes. }\end{array}$ & AUS & CT \\
\hline
\end{tabular}

Ediciones Universidad de Salamanca / CC BY-NC-ND

Siglo Cero, vol. 51 (4), 2020, octubre-diciembre, pp. 69-92 


\section{TABla 4. Características de la selección de documentos (cont.)}

\begin{tabular}{|c|c|c|c|}
\hline Autores, año & Título & País & Met. \\
\hline $\begin{array}{l}\text { Mas, J. M., Cañadas, } \\
\text { M., Balcells-Balcells, A., } \\
\text { Giné, C., Serrano, A. M. y } \\
\text { Dunst, C. J. (2018). }\end{array}$ & $\begin{array}{l}\text { Psychometric properties of the Spanish version } \\
\text { of the family-centred practices scale for use with families } \\
\text { of young children receiving early childhood intervention. }\end{array}$ & ESP & CT \\
\hline $\begin{array}{l}\text { Mas, J. M., Dunst, C. J., } \\
\text { Balcells-Balcells, A., } \\
\text { García-Ventura, S., Giné, } \\
\text { C. y Cañadas, M. (2019). }\end{array}$ & $\begin{array}{l}\text { Family-centered practices and the parental well-being } \\
\text { of young children with disabilities and developmental delay. }\end{array}$ & ESP & CT \\
\hline $\begin{array}{l}\text { Marco, M., Sánchez, M. C. } \\
\text { y García, F. A. (2018). }\end{array}$ & $\begin{array}{l}\text { Inteligencia emocional y prácticas relacionales con familia } \\
\text { en atención temprana. }\end{array}$ & ESP & CT \\
\hline $\begin{array}{l}\text { Martínez, A. y Calet, N. } \\
\text { (2015). }\end{array}$ & $\begin{array}{l}\text { Intervención en Atención Temprana: enfoque desde } \\
\text { el ámbito familiar. }\end{array}$ & ESP & RL \\
\hline $\begin{array}{l}\text { Martínez, M. T. y } \\
\text { Martínez, L. (2013). }\end{array}$ & $\begin{array}{l}\text { Promoción del desarrollo infantil y atención temprana: } \\
\text { calidad de los servicios. }\end{array}$ & ESP & CT \\
\hline $\begin{array}{l}\text { Mayorga, M. J., Madrid, D. } \\
\text { y García, M. P. (2015). }\end{array}$ & $\begin{array}{l}\text { Aprender a trabajar con las familias en Atención Temprana: } \\
\text { estudio de caso. }\end{array}$ & ESP & CT \\
\hline Minard, C. (2018). & $\begin{array}{l}\text { The underutilization of occupational therapy in } \\
\text { transdisciplinary early intervention services. }\end{array}$ & EE. UU. & $\mathrm{RL}$ \\
\hline $\begin{array}{l}\text { Pereira, A. P. S. y Serrano, } \\
\text { A. M. (2014). }\end{array}$ & $\begin{array}{l}\text { Early Intervention in Portugal: study of professionals' } \\
\text { perceptions. }\end{array}$ & PRT & CL \\
\hline $\begin{array}{l}\text { Pighini, M. J., Goelman, H., } \\
\text { Buchanan, M., Schonert- } \\
\text { Reichl, K. y Brynelsen, D. } \\
\text { (2014). }\end{array}$ & $\begin{array}{l}\text { Learning from parents' stories about what works in early } \\
\text { intervention. }\end{array}$ & AUS & CL \\
\hline $\begin{array}{l}\text { Rodger, S., O’Keefe, } \\
\text { A., Cook, M. y Jones, J. } \\
\text { (2012). }\end{array}$ & $\begin{array}{l}\text { Parents' and service providers' perceptions of the family goal } \\
\text { setting tool: a pilot study. }\end{array}$ & AUS & $\mathrm{CL}$ \\
\hline $\begin{array}{l}\text { Romero, R. P., Gálvez, P., } \\
\text { Belda, J. C. y Millá, M. G. } \\
\text { (2015). }\end{array}$ & $\begin{array}{l}\text { Percepción de la calidad en Centros de Atención Temprana: } \\
\text { resultados de un análisis comparativo. }\end{array}$ & ESP & CT \\
\hline Rouse, L. (2012). & $\begin{array}{l}\text { Family-centred practice: empowerment, self-efficacy, } \\
\text { and challenges for practitioners in early childhood } \\
\text { education and care. }\end{array}$ & AUS & RL \\
\hline
\end{tabular}

Nota: Revisión de la Literatura (RL), Cualitativa (CL), Cuantitativa (CT).

Fuente: Elaboración propia.

A partir de la identificación de los clústeres, de las palabras clave de los artículos y de dos lecturas en profundidad, se agruparon los documentos en cuatro categorías relacionales: aspectos generales de la AT (C1), profesionales de la Atención Temprana (C2), familia (C3) y barreras del modelo centrado en la familia (C4). Para un mayor 
análisis de elementos comunes entre los documentos y una adecuada redacción de los resultados se detectaron seis subcategorías: principios y prácticas de la $A T$ (S1C1) - prácticas relacionales y prácticas participativas, principios operacionales y principios conceptuales, principios de ayuda y prácticas de desarrollo de capacidades-, interacción profesional-familia (S1C2) -intervención, relación profesional-familia, pautas, intercambio de información, visitas a domicilio-, equipo de profesionales (S2C2) -equipo interdisciplinar y transdisciplinar y formación profesional-, satisfacción percibida por las familias (S1C3) -satisfacción del servicio y percepción sobre el desarrollo del niño- y barreras del modelo centrado en la familia (S1C4) -barreras del sistema, de los profesionales y de las familias-. En este caso no se tuvo en cuenta el lugar del estudio, debido a que el interés era analizar los factores que implican avances en el campo de la Atención Temprana, así como los que requerían mayor conocimiento e investigación.

Además, se detectó que el 43,3 \% de documentos aportaban contenido a una categoría, mientras que el $50 \%$ a dos categorías y el 16,6 \% a tres. Finalmente, se identificaron los patrones, los hallazgos, las argumentaciones y las contradicciones entre resultados de los estudios seleccionados, haciendo especial hincapié en la justificación de nuevas investigaciones.

\section{Resultados}

\subsection{Principios y prácticas de la Atención Temprana}

Los resultados de la búsqueda demuestran que un $88 \%$ de los artículos seleccionados incluyen familia dentro de la selección de palabras clave. Actualmente, no se concibe contemplar los principios y las prácticas sin hacer mención al ámbito familiar. Además, Epley, Summers y Turnbull (2010) detallan que un 90 \% de los artículos seleccionados para la revisión de la literatura hacen referencia a la relación familiaprofesional como un elemento relevante en las investigaciones. Es más, las prácticas centradas en la familia se vinculan con las percepciones de los familiares sobre el comportamiento de sus hijos, sobre el bienestar familiar y los sentimientos de competencia y autoeficacia de los padres y las madres (Espe-Sherwind, 2008).

La investigación de Mas, Dunst, Balcells-Balcells, García-Ventura, Giné y Cañadas (2019) detalla las características que vertebran las prácticas. Indican que las creencias de los profesionales sobre las fortalezas familiares, la elección informada de la familia, la toma de decisiones y la participación activa de los miembros ayudan a aumentar las capacidades familiares y nuevas habilidades parentales. De acuerdo con Espe-Sherwindt (2008), las prácticas centradas en la familia se estructuran a partir de tres elementos clave: poner énfasis en las fortalezas y no en los déficits, propulsar la importancia de control y la elección familiar sobre los recursos y desarrollar una relación de calidad y colaboración entre padres y profesionales.

Por otro lado, el papel de los profesionales para llevar a cabo las prácticas relacionales y participativas es un componente de elevada trascendencia. Las dos 
tienen importantes consecuencias para el aumento positivo de habilidades parentales (Mas et al., 2019). Sin embargo, las prácticas participativas tienen un valor añadido, ya que no es suficiente ser afectuoso o agradable, sino que se debe establecer una relación de confianza con equilibrios de poder, de manera que las familias decidan sobre el proceso y sean agentes de cambio a partir de sus capacidades (EspeSherwindt, 2008).

Dunst, Espe-Sherwindt y Hamby (2019) constataron, a partir de un estudio de 842 profesionales de los servicios, la evidencia convergente de que las prácticas participativas eran un modelo más adecuado en comparación a las relacionales y que los efectos directos e indirectos sobre el desarrollo de capacidades familiares eran superiores. Los autores determinan que estos factores llevan a una "serie de beneficios positivos para los niños, los padres y la familia” (p. 521).

Por otro lado, Escorcia, García, Sánchez y Hernández (2016) elaboraron un cuestionario ${ }^{2}$ para que los profesionales evaluasen la relación con las familias y, en especial, cómo se llevan a cabo las prácticas relacionales -tercer conjunto de ítems- y las participativas -cuarto conjunto de ítems-. Los resultados obtenidos a partir de las 41 respuestas de los profesionales atribuyeron una clara tendencia por el uso de prácticas relacionales más que participativas. Además, hay estudios que reivindican que, a pesar de no utilizar un modelo plenamente centrado en la familia, siempre se tiene en cuenta la familia como eje transversal del proceso (Marco, Sánchez y García, 2018). Por consiguiente, hay diferentes obstáculos para desarrollar prácticas participativas y no se puede asumir la afirmación de que se efectúan de manera rutinaria en los servicios de Atención Temprana (Mas et al., 2019; Rouse, 2012).

Asimismo, los profesionales identifican una carencia de formación para adquirir estrategias de participación familiar (Dunst et al., 2019; García, Rubio, Orcajada, Escorcia y Cañadas, 2018; Mas, Cañadas, Balcells-Balcells, Giné, Serrano y Dunst, 2018). En efecto, las habilidades participativas son un reto, debido a que la percepción de los profesionales no coincide con la realidad teórica argumentada (Escorcia, García, Sánchez, Orcajada y Hernández, 2018; Espe-Sherwindt, 2008). Solo un estudio meta-analítico demuestra la implicación directa entre las prácticas participativas y los resultados positivos en el desarrollo del niño, lo cual evidencia la falta de investigación en esta área (Gavidia, Meddis y Mahar, 2015).

El siguiente punto a considerar es la implementación de las prácticas centradas en la familia. Los resultados del estudio de Fox, Nordquist, Billen y Savoca (2015), con una muestra de 141 padres, detallan que, a pesar de que el modelo sea centrado en la familia, los padres no perciben ni experimentan su inclusión durante la práctica. Evidencia que también respalda Espe-Sherwind (2008), la cual halla la divergencia entre la investigación teórica y la práctica. Además, existe la necesidad de analizar los programas locales para demostrar las relaciones entre los modelos y los resultados favorables de los niños y la familia (Gavidia et al., 2015), así como estudios enfocados a la práctica profesional y a la evaluación de los programas de Atención Temprana (Epley et al., 2010; Fox et al., 2015).

2 Cuestionario de Estilos de Interacción entre Padres y Profesionales en Atención Temprana (SIPPEI). 


\subsection{Interacción profesional-familia}

Las percepciones de los profesionales sobre su práctica diaria no se corresponden con las valoraciones familiares. El estudio llevado a cabo por Escorcia et al. (2018), a partir de una muestra representativa de 504 familias usuarias y 187 profesionales, determina que las prácticas de relación más comunes son las recomendaciones orales, las sugerencias sobre las rutinas diarias mediante pautas y las recomendaciones por escrito. En cambio, las menos comunes son las visitas a domicilio. Un resultado significativo es que los profesionales exponen que casi siempre cuentan con la familia para realizar actividades con el niño y, por el contrario, las familias explican que casi nunca participan de estas tareas. Adicionalmente, los ítems menos valorados se asocian a las preocupaciones y necesidades familiares, produciéndose una abstención de los profesionales $(42,3 \%)$ en contestar preguntas enfocadas a la construcción conjunta de objetivos. Luego, los autores enfatizan la divergencia entre las acciones profesionales y el uso de buenas prácticas.

Un ejemplo clarificador es que las familias usuarias de los servicios buscan acercamiento, atención y ayuda (Rouse, 2012). Cañadas (2012) manifiesta que los programas con mayor éxito son esos que "ayudan a las familias a ser más competentes y reducen al máximo la participación del profesional” (p. 135). Pighini, Goelman, Buchanan, Schonert y Brynelsen (2014) apuestan por prácticas de naturaleza colaborativa caracterizadas por el seguimiento de las inquietudes de los padres, las solicitudes durante la práctica diaria y la información bidireccional que ayuda a obtener resultados positivos de empoderamiento familiar. Análogamente, diferentes estudios determinan bajas puntuaciones en torno a las percepciones familiares sobre el trato y la interacción recibida por parte de los profesionales (Escorcia et al., 2016; Marco et al., 2018).

Cabe destacar aspectos relacionados con el intercambio de información, debido a que es uno de los pilares básicos de todos los procesos de interacción (Balcells, Giné, Guardia, Summers y Mas, 2019; Escorcia et al., 2018). El cuarto objetivo de la investigación de Martínez y Martínez (2013) demuestra que la información recibida por los padres enfocada a la discapacidad o trastorno de su hijo, así como la evolución sobre su desarrollo, ayuda a que las familias entiendan mejor el proceso. Además, la modalidad de lenguaje que se utilice para fomentar la comunicación se debe adaptar al nivel de comprensión de los progenitores, y el estudio de Marco et al. (2018) refleja que este ítem es el que se identifica con puntuación más baja por parte de los profesionales. Por lo tanto, la transmisión oral no implica que los padres y las madres entiendan ni puedan adoptar estrategias para aplicar las pautas, de manera que es poco probable que las introduzcan a las rutinas diarias (Escorcia et al., 2018).

Por otra parte, la intervención en entornos naturales se caracteriza por el desplazamiento de los profesionales "en los contextos habituales de los niños como el hogar familiar y/o la escuela, para capacitar a los cuidadores informales y poder conseguir una mayor consecución de los objetivos en el desarrollo del niño" (Cañadas, 2012, p. 137). Pues los beneficios son más elevados cuando el profesional accede al contexto natural del niño (Pereira y Serrano, 2014), ya que la comunicación en el 
hogar es más regular y extensa (Fingerhut et al., 2013), la comprensión por parte de los progenitores sobre el trastorno es más profunda y el nivel de comodidad aumenta (Pighini et al., 2014).

Por el contrario, Escorcia et al. (2018) demuestran que nunca o casi nunca los profesionales intervienen en los entornos naturales y remarcan que la mayoría de respuestas en blanco (35,7 \% de familias y $28,9 \%$ de profesionales) se relacionan con la intervención en los contextos naturales. Una posible causa puede ser que no es posible valorar una práctica que no se ha llevado a cabo. Por consiguiente, futuras investigaciones deben explorar las estrategias profesionales en estos ambientes y, sobre todo, indagar sobre la inteligencia emocional de los trabajadores, ya que puede ser un factor condicionante para la práctica profesional en entornos naturales (Marco et al., 2018).

\subsection{Equipo de profesionales}

Los equipos de profesionales que trabajan en los servicios de Atención Temprana deben valorar cada disciplina específica que configura la práctica con el fin de proporcionar una intervención de calidad (Minard, 2018; Pereira y Serrano, 2014). La coordinación entre los miembros de salud, educación y servicios sociales debe ser suficientemente potente, de manera que se asegure un acceso fácil a los servicios por parte de los progenitores (Pereira y Serrano, 2014). Destacados autores indican la necesidad de analizar el modelo organizativo de los servicios, indicando que la gestión, las políticas y las prácticas han de explicar el uso o el no uso del modelo centrado en la familia (Mas et al., 2018). Sin embargo, los profesionales siguen priorizando la cantidad de sesiones sobre la calidad de las intervenciones (Cañadas, 2012). Por ejemplo, García et al. (2018) detallan diferentes conclusiones sobre uno de los servicios sociales de referencia en el Estado español, los CDIAT, y exponen que:

- Las intervenciones se realizan más frecuentemente en el CDIAT (75,2 \%) y, en menor parte, en el domicilio (46,3\%), así como en la escuela $(27,3 \%)$ o en otros contextos naturales $(12,4 \%)$.

- El papel que la familia adquiere durante las intervenciones corresponde un $63,3 \%$ a la presencia y participación, un $47 \%$ a la presencia y a la observación y un $21,5 \%$ no están presentes.

- El número de profesionales que llevan a cabo la intervención corresponde un $59,5 \%$ a un profesional, un $43 \%$ a dos profesionales, un $16,5 \%$ a tres y un $3,3 \%$ a más de tres.

- El conocimiento de los profesionales sobre las prácticas centradas en la familia adquiere 9.10 puntos de importancia otorgada y 8.18 puntos a la necesidad percibida.

Por consiguiente, hablar del papel de los profesionales implica reconocer los diferentes modelos de intervención, aunque la filosofía y los principios básicos deben estar basados en las fortalezas y en las capacidades familiares dentro de un marco ecológico de referencia (Rouse, 2012). En efecto, los profesionales deben trabajar a 
partir de la transdisciplinariedad, es decir, las familias han de recibir el apoyo de un profesional, el cual interviene durante el proceso y consulta sus prácticas con otros miembros del equipo (Minard, 2018). Por lo tanto, supone que el conocimiento y la experiencia se unen para ser aplicados por todos los miembros del equipo (Cumming y Wong, 2012). Evans (2018) expone dos cambios de tareas de un equipo interdisciplinar a uno transdisciplinar:

- Transferencia horizontal de roles: intercambio de tareas entre los profesionales con experiencia similar.

- Transferencia vertical de roles: intercambio de tareas entre profesionales de diferentes disciplinas.

El autor indica que el segundo tipo es la menos deseada, debido a que la capacitación de los profesionales no es equivalente. Además, detalla que es peligroso el intercambio de tareas de experiencia y habilidades del sector de salud -logopedas, terapeutas ocupacionales o fisioterapeutas-. A modo de ejemplo, un trabajador social tiene conocimientos limitados para evaluar el deterioro funcional asociado a una discapacidad. Por ende, dependiendo del caso conviene replantear qué roles se asocian a cada profesional y si conviene intervenir a partir de un equipo interdisciplinar con miembros "de diferentes disciplinas, todos trabajando con la familia" (Evans, 2018, p. 504). Además, aplicar el modelo transdisciplinar no implica, directamente, trabajar con el modelo centrado en la familia, ya que la decisión familiar y la evaluación inicial por parte del equipo pueden reconducir la intervención.

El estudio de Cumming y Wong (2012) muestra que, a pesar de que los profesionales tengan clara la filosofía y el propósito, los conceptos clave para trabajar de manera transdisciplinar no están asentados y, por consiguiente, la intervención no será efectiva. El aspecto que fomenta más barreras es la transmisión del conocimiento práctico por parte de los profesionales de las distintas disciplinas. No es suficiente el contenido, sino que también se requiere de experiencia, instinto y estrategias útiles para atender la situación contextual del niño y la familia. Un impedimento para estos equipos es el tiempo para construir la confianza entre los profesionales, puesto que las organizaciones prevén la duración de la intervención, de manera que se proporcionen servicios de calidad con el menor coste posible (Evans, 2018).

Igualmente, la relación directa entre el trabajo transdisciplinar y las mejoras en el desarrollo de los niños aún no se ha demostrado. Queda por estudiar que esta transferencia sea eficiente y efectiva, así como indagar sobre los beneficios de trabajar transdisciplinariamente en los equipos de profesionales (Cumming y Wong, 2012; Evans, 2018).

Además, se debe incidir en los planes de capacitación profesional que se llevan a cabo en España. Diferentes estudios (Cañadas, 2012; Dias y Cadime, 2019; Escorcia et al., 2018; García et al., 2018; Marco et al., 2018; Mas et al., 2018; Pereira y Serrano, 2014) detallan la necesidad emergente de invertir en formación para poder trabajar a partir de determinadas competencias y actitudes y mejorar la eficiencia de los servicios. Concretamente, García et al. (2018) remarcan la necesidad -8.9 puntos- y la importancia -9.2 puntos- percibida de formación por parte de los profesionales para 
trabajar en equipos transdisciplinares. Asimismo, exponen que no hay diferencias significativas entre los trabajadores noveles -con menos de cuatro años de experiencia-y los expertos, resultado respaldado por Marco et al. (2018). A pesar de esto, manifiestan que la capacitación debe ir enfocada a las estrategias de trabajo y las habilidades emocionales de los profesionales y, en menor parte, a la formación de la disciplina profesional.

\subsection{Satisfacción percibida por las familias}

Conocer las percepciones de los familiares sobre los servicios de Atención Temprana es esencial para poder implementar prácticas de calidad y valorar los cambios políticos y metodológicos necesarios, al igual que mejorar los resultados de los niños y la eficacia de los servicios (Davis y Gavidia, 2009; Dias y Cadime, 2019; Hughes y Gavidia, 2019; Pighini et al., 2014).

La investigación de Romero et al. (2015) muestra una valoración positiva del servicio recibido, enfatizando el resultado de satisfacción sobre el personal especializado -equipo interdisciplinar-y la capacitación profesional. Los familiares tienen en cuenta la coordinación, el seguimiento, la progresión y el plan de intervención como aspectos positivos. Sin embargo, los autores puntualizan que el reto actual es investigar sobre la "gestión de la calidad en los servicios" (p. 74), debido a que se han implementado nuevos programas con distintos financiamientos que requieren la evaluación en todo el Estado español.

Del mismo modo, Martínez y Martínez (2013) examinaron las valoraciones de los padres sobre los CDIAT, obteniendo la máxima puntuación en el ítem referente a la atención personalizada -4.57 sobre $5-$, seguido de la frecuencia de sesiones -3.73 sobre $5-$ y la duración de cada sesión -3.70 sobre 5 -. Además, los progenitores que reciben intervención a partir de un equipo interdisciplinar consideran que la información recibida era de ayuda para atender a sus hijos. Sin embargo, criticaron a la Administración por no dar suficiente apoyo legislativo y económico y demandaron la ampliación de plazas para todas las familias que precisaran estos servicios independientemente del nivel socioeconómico.

Los padres/madres también informan de aspectos negativos tales como problemas de programación, falta de comunicación o la intervención de un solo profesional, ya que identifican que son necesarios otros especialistas (Minard, 2018). Asimismo, hay progenitores que demuestran un grado de satisfacción más elevado con los servicios relacionados directamente con el niño que los relacionados con la familia (Epley et al., 2010; Gavidia et al., 2015) y priorizan el conocimiento de sus derechos para poder atender las necesidades de sus hijos (Hughes y Gavidia, 2019).

Pighini et al. (2014) valoraron la escucha activa y el empoderamiento de una muestra de 11 padres y madres que consideraron la interacción, la coordinación y la práctica conjunta como puntos fuertes del servicio. Sin embargo, el nivel de estrés asociado a la percepción del déficit depende del grado de discapacidad. Esto afecta en la consecución de objetivos y en la eficacia del tratamiento (Botana y Peralbo, 
2014). Adicionalmente, los sentimientos positivos de los padres fomentan creencias positivas y bienestar psicológico, donde el modelo centrado en la familia es el eje de acción relacionado directa e indirectamente con la teoría del empoderamiento formada por modelos que ayudan a desarrollar habilidades y capacidades parentales (Mas et al., 2019).

Adicionalmente, la visión de los padres sobre el desarrollo de sus hijos es un ítem que se debe tener en cuenta para evaluar el grado de satisfacción, ya que "hay diferencias significativas en la asistencia de recursos según el grado de discapacidad” (Gavidia et al., 2015, p. 62). Emerge la clara evidencia de que la gravedad del diagnóstico implica diferente grado de satisfacción. Un ejemplo es que los padres otorgan mayor puntuación al apoyo recibido cuando menor es el grado de discapacidad de su hijo (Barcells, Giné, Guàrdia, Olmos y Summers, 2016; Fox et al., 2015). Sin embargo, solo se ha demostrado la relación indirecta de las prácticas centradas en la familia y los resultados del desarrollo del niño (Hughes y Gavidia, 2019), por lo que "la investigación debe aclarar algunas de las relaciones entre las diferentes y complejas variables de las prácticas de Atención Temprana y los resultados de familia y niño” (Fernández, Serrano, McWilliam y Cañadas, 2017, p. 2).

\subsection{Barreras del modelo centrado en la familia}

Como se ha dicho, la Atención Temprana está pasando por un periodo de cambio, ya que las prácticas profesionales iniciales centradas en el modelo médico intentan dar respuesta al modelo centrado en la familia. Esto ocasiona algunas barreras en la implementación de las prácticas y se asocia directamente a la regulación de la calidad de los servicios (Espe-Sherwindt, 2008).

La primera categoría son las barreras en el sistema. Los profesionales perciben la falta de recursos que afectan al funcionamiento del servicio y la insuficiencia de apoyo por parte de las instituciones, puesto que la normativa que regula la Atención Temprana prioriza la atención enfocada al niño (Cañadas, 2012; Espe-Sherwind, 2008; Fingerhut et al., 2013).

Una segunda categoría está enfocada a la capacitación efectiva de los profesionales. Hasta ahora, la formación se ha caracterizado por el aprendizaje de las disciplinas específicas y, en menor parte, ha ido dirigida a las prácticas participativas que dotan de estrategias para atender a la familia en su contexto (Dias y Cadime, 2019; EspeSherwind, 2008; Pereira y Serrano, 2014). Esto explica las dificultades para trabajar transdisciplinarmente, donde la colaboración o el intercambio de información profesional son elementos que se ven afectados (Pereira y Serrano, 2014). Además, se le añade el factor actitudinal en el momento que los profesionales se consideran expertos, pues la toma de decisiones, el trabajo sobre las capacidades familiares o las visitas en el hogar son aspectos que quedan omitidos (Cañadas, 2012; Dias y Cadime, 2019; Espe-Sherwind, 2008; Fingerhut et al., 2013; Rodger et al., 2012).

La tercera categoría está directamente relacionada con la familia. Las características familiares como los problemas internos, el nivel sociodemográfico y cultural o el 
estado de ánimo contribuyen a que el profesional adopte una actitud de compañerismo más que de intervención. Se debe tener en cuenta que existen familias que no se sienten cómodas con el modelo centrado en la familia, por lo tanto, se debe fomentar la creatividad en los métodos de acceso y colaboración (Finerhut et al., 2013; Pighini et al., 2014). La falta de tiempo, los horarios de trabajo y la periodicidad para ofrecer apoyo total a los progenitores son otro impedimento importante de este modelo (Pereira y Serrano, 2014).

\section{Conclusión}

Los cambios filosóficos, organizativos y estructurales de la Atención Temprana durante las últimas décadas han contribuido a que, hoy en día, el principal modelo de referencia esté basado en la familia (Espe-Sherwind, 2008; Rouse, 2012). Es la apuesta por las fortalezas familiares, la capacitación y las relaciones efectivas entre profesionales y progenitores (Epley, Summers y Turnbull, 2010; Espe-Sherwindt, 2008; Mas et al., 2019) durante la intervención.

Desarrollando prácticas relacionales y participativas, se debe tener en cuenta la familia como eje del proceso (Dunst et al., 2019). Sin embargo, se han hallado obstáculos para ejecutar significativamente prácticas participativas en los servicios de Atención Temprana (Escorcia et al., 2018; Espe-Sherwind, 2008; Marco et al., 2018; Mas et al., 2019; Rouse, 2012) y aún no se ha demostrado una implicación directa con los resultados positivos del desarrollo del niño, lo cual indica la falta de investigación en este ámbito (Fernández et al., 2017; Gavidia et al., 2015; Hughes y Gavidia, 2019).

Paralelamente, se une la carencia de formación de los profesionales para llevar a cabo dicho modelo (Cañadas, 2012; Dias y Cadime, 2019; Dunst et al., 2019; Escorcia et al., 2018; Espe-Sherwind, 2008; Gavidia et al., 2015; Mas et al., 2018; Marco et al., 2018; Pereira y Serrano, 2014). Además, los servicios han de apostar por los principios de calidad (Millá, 2005; Minard, 2018; Pereira y Serrano, 2014) evaluando, constantemente, los programas de intervención (Epley et al., 2010; Fox et al., 2015) a partir de la percepción de los progenitores (Davis y Gavidia, 2009; Dias y Cadime, 2019; Gavidia et al., 2015; Hughes y Gavidia, 2019; Pighini et al., 2014).

En síntesis, pasar de un modelo experto al modelo centrado en la familia no es un reto fácil (Espe-Sherwind, 2008). En este sentido, sería conveniente que las futuras líneas de investigación se centraran en las intervenciones de los equipos de profesionales para valorar el bienestar familiar (Martínez y Calet, 2015), la comparación entre modelos (Epley et al., 2010), el desarrollo de nuevos programas más eficaces (Alcantud-Marín, 2018; Martínez y Calet, 2015) o la actitud de los profesionales para permitir la capacitación familiar (Pereira y Serrano, 2014). Estas lagunas en el ámbito de la Atención Temprana ponen de manifiesto el carácter joven de la disciplina, que debe consolidarse para adecuar la respuesta integral y de calidad a las necesidades de la primera infancia.

Los resultados de este trabajo pretenden aportar evidencias del estado actual de la atención temprana. La revisión de la literatura contribuye a identificar las dimensiones 
que pueden inducir a nuevas investigaciones $y$, al mismo tiempo, conocer aspectos que pueden precisar mejoras. No obstante, se deben tener en cuenta dos limitaciones que se desprenden del estudio. La primera se halla en la diferencia de contextos en que se han llevado a cabo las investigaciones seleccionadas para la revisión. Por este motivo, se ha intentado detallar el tipo de población, la metodología y la muestra de los estudios analizados. Por otro lado, solo se han incluido artículos evaluados por procesos de doble ciego. Este hecho excluye otros documentos científicos como trabajos académicos o capítulos de libro, entre otros.

\section{Referencias bibliográficas}

Alcantud-Marín, F., Pérez-Bou, J. y Alonso-Esteban, Y. (2018). Validez diagnóstica de la escala de desarrollo Merrill Parlmer-R utilizada en la evaluación de acceso a los Centros de Desarrollo Infantil y Atención Temprana. Siglo Cero, 50(1), 53-73. http://dx.doi. org/10.14201/scero20195015373

Balcells-Balcells, A., Giné, C., Guàrdia-Olmos, J. y Summers, J. A. (2016). Proposal of indexes to evaluate family quality of life, partnership, and family support needs. Revista Iberoamericana de Psicología y Salud, 7(1), 31-40. http://dx.doi.org/10.1016/j.rips.2015.10.005

Balcells-Balcells, A., Giné, C., Guàrdia-Olmos, J., Summers, J. A. y Mas, J. M. (2019). Impact of supports and partnership on family quality of life. Research in Developmental Disabilities, 85, 50-60. https://doi.org/10.1016/j.ridd.2018.10.006

Belda, J. C. (2016). Niveles de desarrollo de la Atención Temprana. Revista Española de Discapacidad, 4(1), 219-224. http://dx.doi.org/10.5569/2340-5104.04.01.12

Botana, I. y Peralbo, M. (2014). Familia, estrés y atención temprana. Revista de Estudios e Investigación en Psicología y Educación, 1(1), 55-63. https://doi.org/10.17979/reipe.2014.1.1.23

Bronfenbrenner, U. (1987). La ecología del desarrollo humano. Barcelona: Paidós.

CAÑAdas, M. (2012). La familia, principal protagonista de los centros de desarrollo infantil y Atención Temprana. Edetania: Estudios y Propuestas Socio-Educativas, 41, 129-141. Recuperado de https://bit.ly/36m9Y5b.

CERMI. (2017). Por el derecho primordial de la infancia a la salud y a su pleno desarrollo. Madrid: Grupo Editorial Cinca, S.A. Recuperado de https://bit.ly/2TL5Pow.

Codina, L. (2018). Revisiones bibliográficas sistematizadas. Procedimientos generales y Framework para Ciencias Humanas y Sociales. Barcelona: Universidad Pompeu Fabra, Departamento de Comunicación, Máster Universitario en Comunicación Social. Recuperado de https://bit.ly/2TMdZNB.

Cumming, T. y Wong, S. (2012). Professionals don't play: challenges for early childhood educators working in a transdisciplinary early intervention team. Australian Journal of Early Childhood, 37(1), 127-135. Recuperado de https://bit.ly/35pa500.

Davis, K. y Gavidia-Payne, S. (2009). The impact of child, family, and professional support characteristics on the quality of life in families of young children with disabilities. Journal of Intellectual and Developmental Disability, 34(2), 153-162. https://doi. org/10.1080/13668250902874608

Dempsey, I. y Dunst, C. J. (2004). Helpgiving styles and parent empowerment in families with a young child with a disability. Journal of Intellectual and Developmental Disability, 29(1), 40-51. https://doi.org/10.1080/13668250410001662874 
Dempsey, I. y Keen, D. (2008). A review fo processes and outcomes in family-centered services for children with a disability. Topics in Early Childhood Special Education, 28(1), 42-52. https://doi.org/10.1177/0271121408316699

Dias, P. C. y CADime, I. (2019). Child and family-centered practices in early childhood education and care services: an empirical study with families and practitioners in Portugal. Child and Adolescent Social Work Journal, 36(3), 285-294. https://doi.org/10.1007/s10560-019-00599-7

DíAz, C. (2019). Guía básica sobre Atención Temprana y Transformación. Madrid: Plena inclusión España. Recuperado de https://bit.ly/2tFFMVc.

Dunst, C. J. (2000). Revisiting "Rethinking Early Intervention". Topics in Early Childhood Special Education, 20(2), 95-104. https://doi.org/10.1177/027112140002000205

Dunst, C. J. y Bruder, M. B. (2002). Valued outcomes of service coordination, early intervention, and natural environments. Exceptional Children, 68, 361-375. https://doi. org $/ 10.1177 / 001440290206800305$

Dunst, C. J., Espe-Sherwindt, M. y Hamby, D. W. (2019). Does capacity-building professional development engender practitioners' use of capacity-building family-centered practices? European Journal of Educational Research, 8(2), 515-526. http://doi.org/10.12973/eu-jer.8.2.513

Dunst, C. J. y Trivette, C. M. (2009). Capacity-building family-systems intervention practices. Journal of Family Social Work, 12(2), 119-143. https://doi.org/10.1080/10522150802713322

Epley, P., Summers, J. A. y Turnbull, A. (2010). Characteristics and trends in familycentered conceptualizations. Journal of Family Social Work, 13(3), 269-285. https://doi. org/10.1080/10522150903514017

Escorcia-Mora, C. T., García-Sánchez, F. A., Sánchez-López, M. C. y HernándezPÉrez, E. (2016). Styles questionnaire of interaction between parents and practitioners in early intervention: content validity. Anales de Psicología, 32(1), 148-157. Recuperado de https://bit.ly/2Gbk5yK.

Escorcia-Mora, C. T., García-Sánchez, F. A., Sánchez-López, M. C., Orcajada, N. y Hernández-PÉrez, E. (2018). Early childhood intervention practices in the southeast of Spain: Professionals and families perspective. Anales de Psicologia, 34(3), 500-509. https:// doi.org/10.6018/analesps.34.3.311221

ESPE-SHERWINDT, M. (2008). Family-centred practice: collaboration, competency and evidence. Support for Learning, 23(3), 136-143. https://doi.org/10.1111/j.1467-9604.2008.00384.x.

Evans, S. (2017). The social worker as transdisciplinary professional: a reflective account. Australian Social Work, 70(4), 500-507. https://doi.org/10.1080/0312407X.2016.1265997

Fernández, R., Serrano, A. M., McWilliam, R. y Cañadas, M. (2017). Relación entre empoderamiento familiar y calidad de los servicios de atención temprana. Revista de Estudios e Investigación en Psicología y Educación, 11. https://doi.org/10.17979/reipe.2017.0.11.3054

Fingerhut, P. E., Piro, J., Sutton, A., Campbell, R., Lewis, C., Lawji, D. y Martínez, N. (2013). Family-centered principles implemented in home-based, clinic-based, and schoolbased pediatric settings. American Journal of Occupational Therapy, 67(2), 228-235. https:// doi.org/10.5014/ajot.2013.006957

Fox, G. L., Nordquist, V. M., Billen, R. M. y Savoca, E. F. (2015). Father involvement and early intervention: effects of empowerment and father role identity. Family Relations, 64(4), 461-475. https://doi.org/10.1111/fare.12156

García-Sánchez, F. A., Rubio-Gómez, N., Orcajada-Sánchez, N., Escorcia-Mora, C. T. y Cañadas, M. (2018). Training needs in family-centered practices for Spanish early childhood intervention professionals. Bordón, Revista de Pedagogia, 70(2), 39-55. https:// doi.org/10.13042/Bordon.2018.59913 
GAT. (2000). Libro Blanco de la Atención Temprana. Madrid: Real Patronato sobre Discapacidad. Recuperado de https://bit.ly/2sMJLPn.

GAT. (2005). Recomendaciones Técnicas para el desarrollo de la Atención Temprana. Madrid: Real Patronato sobre Discapacidad. Recuperado de https://bit.ly/2Gdxmae.

GAT. (2018). Atención Temprana la visión de los profesionales. Recuperado de https://bit. ly/38sXSs9.

Gavidia-Payne, S., Meddis, K. y Mahar, N. (2015). Correlates of child and family outcomes in an Australian communitybased early childhood intervention program. Journal of Intellectual and Developmental Disability, 40(1), 57-67. http://dx.doi.org/10.3109/13668250.2 014.983056

Giné, C., Gràcia, M., Vilaseca, R. y Balcells, A. (2008). Trabajar con las familias en atención temprana. Revista Interuniversitaria de Formación del Profesorado, 65(23), 95-113. Recuperado de https://bit.ly/37ho9tr.

Gútiez, P. (2005). Conceptualización de la Atención Temprana. En M. G. Millá y F. Mulas (Eds.), Atención Temprana. Desarrollo infantil, diagnóstico, trastornos e intervención. Valencia, España: Promolibro.

Hart, C. (2008). Doing a literature review. Realising the Social Science research imagination. Londres: Sage Publications.

Hughes-Scholes, C. H. y Gavidia-Payne, S. (2019). Early childhood intervention program quality: examining family-centered practice, parental self-efficacy and child and family outcomes. Early Childhood Education Journal, 47(6), 719-729. https://doi.org/10.1007/ s10643-019-00961-5

LeAL, L. (2008). Un enfoque de la discapacidad intelectual centrado en la familia. Madrid: FEAPS Confederación Española de Organizaciones a favor de las Personas con Discapacidad Intelectual. Recuperado de https://bit.ly/3axA1JP.

Marco, M., Sánchez, M. C. y García, F. A. (2018). Inteligencia emocional y prácticas relacionales con familia en atención temprana. Siglo Cero, 49(2), 7-25. http://dx.doi.org/10.14201/ scero2018492725

Martínez, A. y Calet, N. (2015). Intervención en Atención Temprana: enfoque desde el ámbito familiar. Escritos de Psicología, 8(2), 33-42. https://doi.org/10.5231/psy.writ.2015.1905

Martínez, M. T. y Martínez, L. (2013). Promoción del desarrollo infantil y atención temprana: calidad de los servicios. Revista Internacional de Trabajo Social y Bienestar, 2, 49-67. Recuperado de https://bit.ly/2YNshxV.

Mas, J. M., Cañadas, M., Balcells-Balcells, A., Giné, C., Serrano, A. M. y Dunst, C. J. (2018). Psychometric properties of the Spanish version of the family-centred practices scale for use with families of young children receiving early childhood intervention. Journal of Applied Research in Intellectual Disabilities, 31(5), 851-861. https://doi.org/10.1111/ jar.12442.

Mas, J. M., Dunst, C. J., Balcells-Balcells, A., García-Ventura, S., Giné, C. y Cañadas, M. (2019). Family-centered practices and the parental well-being of young children with disabilities and developmental delay. Research in Developmental Disabilities, 94. https:// doi.org/10.1016/j.ridd.2019.103495

McWilliam, R. A. (2010). Routines-based Early Intervention: supporting young children and their families. Baltimore: Paul H. Brookes.

Millá, M. G. (2005). Reseña histórica de la Atención Temprana. En M. G. Millá y F. Mulas (Eds.), Atención Temprana. Desarrollo infantil, diagnóstico, trastornos e intervención. Valencia, España: Promolibro. 
MinaRd, C. (2018). The underutilization of occupational therapy in transdisciplinary early intervention services. Journal of Occupational Therapy, Schools, and Early Intervention, 11(1), 15-20. https://doi.org/10.1080/19411243.2017.1408441

ONU, Asamblea General. (1989) Convención sobre los Derechos del Niño. Resolución 44/25, de 20 de noviembre de 1989, United Nations, Treaty Series, vol. 1577. Recuperado de https://www.unicef.org/convencion(5).pdf.

Pereira, A. P. S. y Serrano, A. M. (2014). Early Intervention in Portugal: study of professionals' perceptions. Journal of Family Social Work, 17(3), 263-282. https://doi.org/10.1080/ 10522158.2013.865426

Pighini, M. J., Goelman, H., Buchanan, M., Schonert-Reichl, K. y Brynelsen, D. (2014). Learning from parents' stories about what works in early intervention. International Journal of Psychology, 49(4), 263-270. https://doi.org/10.1002/ijop.12024

Ponte, J., Cardama, J., Arlanzón, J. L., Belda, J. C., González, T. y Vived, E. (2004). Guía de estándares de calidad en atención temprana. Madrid: IMSERSO. Recuperado de https:// bit.ly/2Rgbb9L.

Rodger, S., O’Keefe, A., Cook, M. y Jones, J. (2012). Parents' and Service Providers' Perceptions of the family goal setting tool: a pilot study. Journal of Applied Research in Intellectual Disabilities, 25(4), 360-371. https://doi.org/10.1111/j.1468-3148.2011.00674.x

Romero, R. P., Gálvez, P., Belda, J. C. y Millá, M. G. (2015). Percepción de la calidad en Centros de Atención Temprana: Resultados de un análisis comparativo. Escritos de Psicología, 8(2), 71-76. https://10.5231/psy.writ.2015.1206.

Romero, R. P., Lillo, M. y García, S. (2018). Calidad percibida en Centros de Atención Temprana de Andalucía. Estudio cualitativo. Siglo Cero, 50(2), 7-18. http://dx.doi. org/10.14201/scero2019502718

Rouse, L. (2012). Family-centred practice: empowerment, self-efficacy, and challenges for practitioners in early childhood education and care. Contemporary Issues in Early Childhood, 13(1), 17-26. http://dx.doi.org/10.2304/ciec.2012.13.1.17

Sameroff, A. J. y Fiese, B. H. (2000). Models of development and developmental risk. En C. H. Zeanah (Ed.), Handbook of infant mental health (2. ${ }^{a}$ ed., pp. 3-19). New York: Guilford Press.

TAMARIT, J. (2015). La transformación de los servicios hacia la calidad de vida. Una iniciativa de innovación social de FEAPS. Siglo Cero, 46(3), 47-71. http://dx.doi.org/10.14201/scero20154634771

Trivette, C. M. y Dunst, C. J. (2004). Evaluating family-based practices: Parenting Experiences Scale. Young Exceptional Children, 7(3), 12-19. https://doi.org/10.1177/109625060400700302

Trivette, C. M., Dunst, C. J. y Hamby, D. W. (2010). Influences of family-systems intervention practices on parent-child interactions and child development. Topics in Early Childhood Special Education, 30, 3-19. https://doi.org/10.1177/0271121410364250 